\title{
THE TORSION PONTRYAGIN CLASSES
}

\author{
EMERY THOMAS ${ }^{1}$
}

Let $\eta$ be a real $n$-plane bundle over a paracompact space $B$. With $\eta$ one associates two (total) characteristic classes: the Stiefel-Whitney class

$$
W(\eta)=1+W_{1}(\eta)+W_{2}(\eta)+\cdots+W_{n}(\eta) \quad\left(W_{i}(\eta) \in H^{i}\left(B ; Z_{2}\right)\right)
$$

and the Pontryagin class

$$
p(\eta)=1+p_{1}(\eta)+\cdots+p_{q}(\eta) \quad\left(p_{j}(\eta) \in H^{4 j}(B ; Z)\right) .
$$

Here $Z=$ integers, $Z_{2}=$ integers $\bmod 2$, and $q=[n / 2]$-the largest integer in $n / 2$.

Recall that the homogeneous Pontryagin classes, $p_{i}(\eta)$, are defined by

$$
p_{i}(\eta)=(-1)^{i} c_{2 i}\left(\eta_{c}\right) \quad(1 \leqq i \leqq[n / 2]),
$$

where $\eta_{c}$ is the complexification of the bundle $\eta$ and $c_{j}\left(\eta_{c}\right)$ denotes the $j$ th Chern class of the complex $n$-plane bundle $\eta_{c}$ (see [1]).

For convenience of notation set

$$
P_{2 k}(\eta)=(-1)^{q} c_{k}\left(\eta_{c}\right),
$$

where $q=[k / 2]$ and $1 \leqq k \leqq 2 n$. Thus, $p_{j}(\eta)=P_{4 j}(\eta)$. We call the classes $P_{4 k+2}(\eta)(k \geqq 0)$ the torsion Pontryagin classes of $\eta$, since [1] $2 P_{4 k+2}(\eta)$ $=0$. These torsion classes occur in an important situation-that is, in the expression for the Pontryagin classes of the (Whitney) sum of two bundles. The purpose of this note is to express these torsion classes in terms of the standard characteristic classes of the bundle $\eta$. In fact, we give three equivalent expressions for them!

Theorem. Let $\eta$ be a real $n$-plane bundle. Then, for $k \geqq 0$,

$$
\begin{aligned}
P_{4 k+2}(\eta) & =\delta_{*}\left(W_{2 k}(\eta) W_{2 k+1}(\eta)\right)=p_{k}(\eta) \delta_{*} W_{1}(\eta)+\left(\delta_{*} W_{2 k}(\eta)\right)^{2} \\
& =\delta_{*} S q^{2 k} W_{2 k+1}(\eta) .
\end{aligned}
$$

Here $\delta_{*}$ is the Bockstein coboundary associated with the exact sequence

$$
0 \rightarrow Z \stackrel{2}{\rightarrow} Z \rightarrow Z_{2} \rightarrow 0
$$

and $S q^{2 k}$ is the Steenrod cohomology operator.

Received by the editors April 25, 1961.

${ }^{1}$ Research supported in part by U. S. Air Force contract 49(638)-79. 
By the usual naturality argument it suffices to prove the theorem for the case $\eta=\gamma^{n}$, the classifying bundle over the real Grassmann complex $G_{n}$-that is, the space whose points are all $n$-dimensional linear subspaces of $R^{\infty}$ ( $R=$ real numbers; see [1] for details).

Denote by $P^{\infty}$ the real, infinite-dimensional projective space $\left(P^{\infty}=G_{1}\right)$ and by $\gamma_{i}^{1}(1 \leqq i \leqq n)$ the 1 -plane bundle over the $n$-fold Cartesian product $\left(P^{\infty}\right)^{n}$ induced from $\gamma^{1}$ by the $i$ th projection map $\left(P^{\infty}\right)^{n} \rightarrow P^{\infty}$. Set

$$
\xi^{n}=\gamma_{1}^{1} \oplus \cdots \oplus \gamma_{n}^{1} \quad(\oplus=\text { Whitney sum }) .
$$

By the same argument as that used to prove Theorem 5.1 of [2], one obtains

$$
\rho_{2} P_{4 k+2}\left(\xi^{n}\right)=W_{2 k+1}\left(\xi^{n}\right)^{2},
$$

where $\rho_{r}$ is induced by the factor homomorphism $Z \rightarrow Z_{r}(r=2,3, \cdots)$.

Let $f$ be a map from $\left(P^{\infty}\right)^{n}$ to $G_{n}$ which induces $\xi^{n}$ from $\gamma^{n}$. Since $f_{2}^{*}$, the $\bmod 2$ cohomology homomorphism induced by $f$, is a monomorphism we obtain-by naturality of the characteristic classes,

$$
\rho_{2} P_{4 k+2}\left(\gamma^{n}\right)=W_{2 k+1}\left(\gamma^{n}\right)^{2} .
$$

Denote by $T$ the torsion subgroup of $H^{*}\left(G_{n} ; Z\right)$. Since every element in $T$ has order 2 it follows that two classes, $x$ and $y$, in $T$ are equal if, and only if, $\rho_{2}(x)=\rho_{2}(y)$. Now, as remarked above, $P_{\mathbf{4 k + 2}}\left(\gamma^{n}\right)$ $\in T$. Therefore, the first equality in our theorem follows from (1) when we note that

$$
\begin{aligned}
\rho_{2} \delta_{*}\left(W_{2 k} W_{2 k+1}\right) & =\beta_{2}\left(W_{2 k} W_{2 k+1}\right) \\
& =\left(W_{2 k+1}+W_{1} W_{2 k}\right)\left(W_{2 k+1}\right)+W_{2 k}\left(W_{1} W_{2 k+1}\right) \\
& =W_{2 k+1}^{2} .
\end{aligned}
$$

Here $W_{i}=W_{i}\left(\gamma^{n}\right)$, and $\beta_{2}$ is the derivation $\rho_{2} \delta_{*}$ which has the values

$$
\begin{aligned}
\beta_{2} W_{2 i}(\eta) & =W_{1}(\eta) W_{2 i}(\eta)+W_{2 i+1}(\eta), \\
\beta_{2} W_{2 i-1}(\eta) & =W_{1}(\eta) W_{2 i-1}(\eta)
\end{aligned} \quad(i \geqq 1),
$$

for any bundle $\eta$.

The second equality in the theorem follows likewise from (1):

$$
\begin{aligned}
\rho_{2}\left(p_{k} \delta_{*} W_{1}+\left(\delta_{*} W_{2 k}\right)^{2}\right) & =\left(\rho_{2} p_{k}\right)\left(\rho_{2} \delta_{*} W_{1}\right)+\left(\rho_{2} \delta_{*} W_{2 k}\right)^{2} \\
& =\left(\rho_{2} p_{k}\right)\left(\beta_{2} W_{1}\right)+\left(\beta_{2} W_{2 k}\right)^{2} \\
& =\left(W_{2 k}\right)^{2}\left(W_{1}\right)^{2}+\left(W_{1} W_{2 k}+W_{2 k+1}\right)^{2} \\
& =\left(W_{2 k+1}\right)^{2}=\rho_{2} P_{4 k+2} .
\end{aligned}
$$


Here we have used the fact that $\rho_{2}$ is a multiplicative homomorphism and that $\rho_{2} p_{k}=W_{2 k}^{2}\left(p_{k}=p_{k}\left(\gamma^{n}\right)\right)$.

Finally, to obtain the third equality notice that

$$
\rho_{2} \delta_{*} S q^{2 k}=\beta_{2} S q^{2 k}=S q^{1} S q^{2 k}=S q^{2 k+1} .
$$

Since $W_{2 k+1}$ is a $(2 k+1)$-dimensional cohomology class,

$$
S q^{2 k+1} W_{2 k+1}=\left(W_{2 k+1}\right)^{2},
$$

and therefore

$$
\rho_{2} \delta * S q^{2 k} W_{2 k+1}=\left(W_{2 k+1}\right)^{2}=\rho_{2} P_{4 k+2},
$$

completing the proof of the theorem.

Suppose now that $\eta$ and $\zeta$ are two bundles over the same base space. Define for $k \geqq 1$,

$$
D_{k}(\eta, \zeta)=p_{k}(\eta \oplus \zeta)-\sum_{i+j=k} p_{i}(\eta) p_{j}(\zeta) ;
$$

that is, $D_{k}$ measures the deviation of the Pontryagin class $p_{k}$ from following the multiplicative rule for characteristic classes. Using the fact that $(\eta \oplus \zeta)_{c}$ is equivalent to $\eta_{c} \oplus \zeta_{c}$, together with the multiplicative property of the Chern class, one has

$$
P_{4 k}(\eta \oplus \zeta)=\sum_{i+j=2 k} P_{2 i}(\eta) P_{2 j}(\zeta) \text {. }
$$

Thus, using the first equality in the theorem, we obtain: ${ }^{2}$

(2) Let $\eta, \zeta$ be bundles over the same base space. Then, for $k \geqq 1$,

$$
D_{k}(\eta, \zeta)=\sum_{i+j=k-1} \delta_{*}\left[W_{2 i}(\eta) W_{2 i+1}(\eta)\right] \delta_{*}\left[W_{2 j}(\zeta) W_{2 j+1}(\zeta)\right]
$$

Suppose that $\eta$ and $\zeta$ are both orientable bundles. Then, $W_{1}(\eta)=$ $W_{1}(\zeta)=0$, and consequently,

$$
D_{1}(\eta, \zeta)=D_{2}(\eta, \zeta)=0
$$

Continuing in this fashion, recall that an $n$-plane bundle $\xi$ is equivalent to a bundle with $\operatorname{Spin}(n)$ as structure group if, and only if, $W_{1}(\xi)=W_{2}(\xi)=0$. Denote by $B_{\operatorname{Spin}(n)}$ a classifying space for $\operatorname{Spin}(n)-$ bundles. Then one easily shows that

$$
H^{i}\left(B_{\mathrm{Spin}(n)} ; Z\right)=0, \quad 1 \leqq i \leqq 3 ; \quad H^{4}\left(B_{\operatorname{Spin}(n)} ; Z\right) \text { is free abelian. }
$$

Thus for a $\operatorname{Spin}(n)$-bundle $\xi$,

2 Cartan notes an equivalent formula in Expose 17 (equation no. 59') of Séminaire H. Cartan 1959/1960, Paris. 


$$
W_{1}(\xi)=W_{3}(\xi)=W_{5}(\xi)=0 .
$$

Applying this information to equation (2) we obtain:

(3) Let $\eta, \zeta$ be orientable bundles over the same base and suppose that

$$
W_{2}(\eta)=W_{2}(\zeta)=0 .
$$

Then,

$$
D_{k}(\eta, \zeta)=0, \quad \text { for } 1 \leqq k \leqq 6 .
$$

There is one other place where the torsion Pontryagin classes play a role. Denote by $\mathfrak{B}_{2}$ the Pontryagin square cohomology operation and by $\theta_{2}$ the cohomology homomorphism induced by the inclusion $Z_{2} \rightarrow Z_{4}$. Then, $W u$ has shown (see $[2 ; 3]$ ) that

$$
\mathfrak{P}_{2}\left(W_{2 i+1}(\eta)\right)=\rho_{4} \delta_{*} S q^{2 i} W_{2 i+1}(\eta)+\theta_{2}\left(W_{1}(\eta) S q^{2 i} W_{2 i+1}(\eta)\right),
$$

where $\eta$ is any real $n$-plane bundle. Thus, by the third equality of our theorem we obtain:

(4) Let $\eta$ be an $n$-plane bundle. Then, for $i \geqq 0$,

$$
\mathfrak{P}_{2}\left(W_{2 i+1}(\eta)\right)=\rho_{4} P_{4 i+2}(\eta)+\theta_{2}\left(W_{1}(\eta) S q^{2 i} W_{2 i+1}(\eta)\right) .
$$

\section{BIBLIOGRAPHY}

1. J. Milnor, Lectures on characteristic classes, mimeographed notes, Princeton University, 1957.

2. E. Thomas, On the cohomology of the real Grassmann complexes..., Trans. Amer. Math. Soc. 96 (1960), 67-89.

3. W. Wu, On Pontryagin classes. III, Acta Math. Sinica 4 (1954), 323-347.

University of California at Berkeley 